\title{
A Case Report of Ablepharon- Macrostomia Syndrome with Amniotic Membrane Grafting
}

\author{
Eric Feinstein $^{\mathrm{a}} \quad$ Aisha S. Traish $^{\mathrm{a}}$ Vinay Aakalu $^{\mathrm{a}} \quad$ Iris S. Kassem ${ }^{\mathrm{a}, \mathrm{b}}$ \\ ${ }^{a}$ Department of Ophthalmology and Visual Sciences, University of Illinois at Chicago \\ College of Medicine, Chicago, Ill., and ${ }^{\mathrm{b}}$ Department of Ophthalmology, Medical College of \\ Wisconsin Eye Institute, Milwaukee, Wisc., USA
}

\section{Key Words}

Ablepharon · Macrostomia · Congenital eyelid anomalies · Absent hair · Absent eyelids

\begin{abstract}
We describe a rare case of an infant who was born with multiple congenital anomalies, including the absence of eyelids. This patient had many dysmorphic features consistent with a severe phenotype of ablepharon-macrostomia syndrome (AMS) including a fish-like appearance of the mouth, rudimentary ears, absence of body hair, thin skin, absent nipples, abdominal distension, and genital abnormalities. Upon presentation, there was severe exposure keratopathy causing large bilateral sterile ulcers culminating in corneal melting of both eyes. An amniotic membrane graft was used to attempt to maintain the corneal surface integrity. However, because of the late presentation, the corneas could not be salvaged. Extensive surgical reconstruction of both eyelids and bilateral penetrating keratoplasty was ultimately performed successfully to protect the ocular surfaces while trying to maximize the visual potential. Early amniotic membrane grafting may be done at the bedside and may help preserve the ocular in patients with severe eyelid deformities until more definitive treatment is performed.

\section{Case Report}

A 5-day-old female infant was transferred from an outside hospital for further ophthalmic and neonatal care. Prenatal history was significant for poor prenatal care and alcohol use during pregnancy. She was born at 37 weeks without complications. Birth weight was in

KARGER 125:s $\quad \begin{aligned} & \text { Iris S. Kassem, MD, PhD } \\ & \text { Department of Ophthalmology } \\ & \text { Medical College of Wisconsin } \\ & 925 \text { N. 87th Street, Milwaukee, WI } 53226 \text { (USA) } \\ & \text { E-Mail ikassem@mcw.edu }\end{aligned}$


Feinstein et al.: A Case Report of Ablepharon-Macrostomia Syndrome with Amniotic Membrane Grafting

the 3rd percentile and multiple severe malformed features were noted (fig. 1a, b). Multiple ocular anomalies were seen on initial exam including, but not limited to, ablepharon with small corneal diameters and corneal exposure (fig. 1b). Other facial abnormalities were a sloped forehead, hypertelorism, flattened nasal bridge, macrostomia, micrognathia, deformed ear lobes, thin upper lip with a long philtrum, and reduced hair of the scalp and eyebrows (fig. 1a). She also had absent nipples, abnormal skin tension throughout her entire body with absence of hair or lanugo, and abnormal genitalia.

There was no family history of maternal genetic or ocular disorders, but the paternal history revealed a report of a prior child by a different partner with congenital heart disease and unspecified absence of part of one arm. Systemic workup including echocardiogram showed a patent foramen ovale with normal ventricles, atria, valves and systolic and diastolic function. Abdominal (kidneys, spleen, and bladder) and head ultrasounds were normal. A genetics workup showed normal standard chromosomal analysis except a normal variant of the 9p12q13 mutation observed in every cell. Chromosomal microarray and karyotype were normal.

Upon initial ophthalmic examination, a typical aversion to light of either eye was found. There was full extraocular motility, and intraocular pressures were $10 \mathrm{~mm}$ Hg measured with a Tonopen (Reichert, Depew, N.Y., USA). On external examination, we observed an absence of eyelids, eyelashes, and eyebrows with scant orbicularis muscle present with retraction of the globe when blinking (fig. 1b). There was marked injection and chemosis of the conjunctiva in both eyes. The cornea was found to have an epithelial defect of 5 and $6 \mathrm{~mm}$ OD and OS, respectively, and bilateral corneal opacification and $60 \%$ thinning in both eyes. Corneal diameters were $7 \mathrm{~mm}$ OD and $8 \mathrm{~mm}$ OS. There was no view to the anterior chamber, iris or lens. B-scan ultrasonography did not reveal any posterior segment abnormalities. Aerobic, anaerobic, fungal and acid-fast bacillus cultures of the cornea were negative with no organisms seen on Gram stain. Cryopreserved amniotic membranes were placed across both corneas at the bedside.

Despite aggressive lubrication, there was migration of the amniotic membrane followed by progressive thinning of the corneas noted with eventual full-thickness melting in both eyes (fig. 1c). The patient was admitted for immediate penetrating keratoplasty in both eyes as well as full-thickness reconstruction of the eyelids, including extensive conjunctivoplasty with reconstruction of the fornix, full-thickness skin graft for upper lids from postauricular area and temporary tarsorrhaphy in both eyes (fig. 1d). Pathology of both corneas with Gram stain and Giemsa staining confirmed a sterile keratopathy (fig. 2).

After 1 week, the patient was noted to have flattening of the anterior chamber and a wound leak OS, and an immediate wound revision was performed. Her follow-up exam at 5 and a half months showed that she was able to track light binocularly. She had a mild haze of her right corneal graft and mild conjunctivalization of the left graft. However, she had sufficient eyelid with Bell's palsy to keep the globes hydrated with lubricant supplementation (fig. 3). Systemically, the patient could not thrive properly with weight loss requiring the placement of a gastric tube.

\section{Discussion}

AMS is an extremely rare disorder first described by McCarthy and West [1] in 1977 with the most common features being absence of eyelids, sparse or absence of hair, a large fish-like mouth (macrostomia), ear and genital anomalies, and redundant coarse skin [1-4]. As of 2011, fewer than 20 cases of AMS had been reported in the literature worldwide [2]. In 
Feinstein et al.: A Case Report of Ablepharon-Macrostomia Syndrome with Amniotic Membrane Grafting

the vast majority of these patients, the chromosomal studies were normal $[3,4]$. A possible mutation on chromosome 18 has been reported [5]. A de novo alteration of the TWIST2 gene, which is located on chromosome 2 and involved in mesenchymal cell differentiation, has recently been identified [6]. Our patient had a 9p12q13 chromosome inversion mutation observed in every cell of unknown significance. Her mother had a history of alcohol use during pregnancy, which may or may not have contributed to this syndrome.

There are variable severities of AMS. Stevens and Sargent [3] reported 3 cases of AMS as well as a 10-year follow-up on a previously reported patient. These patients had similar findings to our patient including low birth weight, absence of the eyelids, sparse or absent hair (eyebrows/eyelashes), macrostomia, ear anomalies, abnormal genetalia, redundant skin, and abnormal ears. Two out of the 3 cases had significant chemosis and corneal exposure keratopathy leading to significantly reduced vision $(<20 / 400)$, similar to our patient. The 10 -year-old child and the 27-year-old adult in this case series with AMS both had significant corneal exposure at birth and underwent eyelid surgery followed by skin grafting for eyelid reconstruction within the first weeks of life. Brancati et al. [7] found similar findings in a 46year-old female. The case we report here seems to be a severe phenotype when in comparison to previous reports of AMS and is the first to describe the microcornea phenotype.

In this report, we present a new case of AMS with a unique treatment of corneal exposure with amniotic membrane grafting not available at the time of the other reports. Our patient had significant ocular complications related to exposure that may have been prevented with earlier intervention. We highlight the importance of early treatment and potential new avenues for intervention, while recognizing the difficulties that can be encountered when treating patients with severe exposure due to eyelid abnormalities. In this case, we placed an amniotic membrane graft, which maintained the corneal surface for a brief period of time. Patients such as these may benefit from immediate placement of a large amniotic graft allowing more time for the coordination of a multispecialty team for the upcoming intervention or for the organization of the transportation to another hospital. A hard eye shield should also be placed over each eye to prevent contact of the corneal surface. A similar technique has been described in an infant with partial cryptophthalmos [8]. The advantage of an amniotic membrane graft is that it can be placed at the bedside, as was done in this case. This eliminates the need for anesthesia or the use of an operating room in cases where it may not be available. Without eyelids and associated meibomian glands, the risk of corneal decompensation from severe exposure keratopathy may occur rapidly. The importance of temporary temporizing measures with eye lubricants and amniotic membrane grafting while arranging for a more permanent solution such as eyelid reconstruction will help to preserve corneal integrity and improve the likelihood of achieving maximal visual potential. In our patient, because of the extreme desiccation and thinning of the corneas at presentation, corneal melting still occurred with intensive care. A corneal transplant was inevitable in order to preserve the eye in the hopes that visual rehabilitation could follow. The postoperative wound integrity was also compromised because of the friability of the cornea. However, this may have extended the time until the patient needed surgery. In less severe cases of eyelid abnormalities, amniotic membrane grafting may be a viable alternative that may prevent corneal melting secondary to exposure.

\section{Acknowledgements}

This work was supported by an unrestricted grant from NIH grant No. K08 EY024645 and a departmental unrestricted grant from the Research to Prevent Blindness. 
Feinstein et al.: A Case Report of Ablepharon-Macrostomia Syndrome with Amniotic Membrane Grafting

\section{Statement of Ethics}

The guardian of the patient presented in this case has given written consent for the publication of the photographies.

\section{Disclosure Statement}

The authors declare that there are no conflicts of interest.

\section{References}

1 McCarthy GT, West CM: Ablepheron macrostomia syndrome. Dev Med Child Neurol 1977;19:659-672

-2 Kallish S, McDonald-McGinn DM, van Haelst MM, Bartlett SP, Katowitz JA, Zackai EH: AblepharonMacrostomia syndrome - Extension of the phenotype. Am J Med Genet A 2011;155A:3060-3062.

-3 Stevens CA, Sargent LA: Ablepharon-macrostomia syndrome. Am J Med Genet 2002;107:30-37.

4 Jackson IT, Shaw KE, del Pinal Matorras F: A new feature of the ablepharon macrostomia syndrome: zygomatic arch absence. Br J Plast Surg 1988;41:410-416.

-5 Pellegrino JE, Schnur RE, Boghosian-Sell L, Strathdee G, Overhauser J, Spinner NB, Stump T, Grace K, Zackai EH: Ablepharon macrostomia syndrome with associated cutis laxa: possible localization to 18q. Hum Genet 1996;97:532-536.

6 Marchegiani S, Davis T, Tessadori F, et al: Recurrent mutations in the basic domain of TWIST2 cause ablepharon macrostomia and Barber-Say syndromes. Am J Hum Genet 2015;97:99-110.

-7 Brancati F, Mingarelli R, Sarkozy A, Dallapiccola B: Ablepharon-macrostomia syndrome in a 46-year-old woman. Am J Med Genet 2004;127A:96-98.

8 Murthy R, Gupta H: Novel surgical technique for the management of partial cryptophthalmos. Indian J Ophthalmol 2014;62:1096-1098. 


\section{Case Reports in \\ Ophthalmology}

\begin{tabular}{l|l}
\hline Case Rep Ophthalmol 2015;6:366-372 \\
\hline DOI: 10.1159/000441615 & $\begin{array}{l}\text { ○ 2015 The Author(s). Published by S. Karger AG, Basel } \\
\text { www.karger.com/cop }\end{array}$ \\
\hline
\end{tabular}

Feinstein et al.: A Case Report of Ablepharon-Macrostomia Syndrome with Amniotic Membrane Grafting
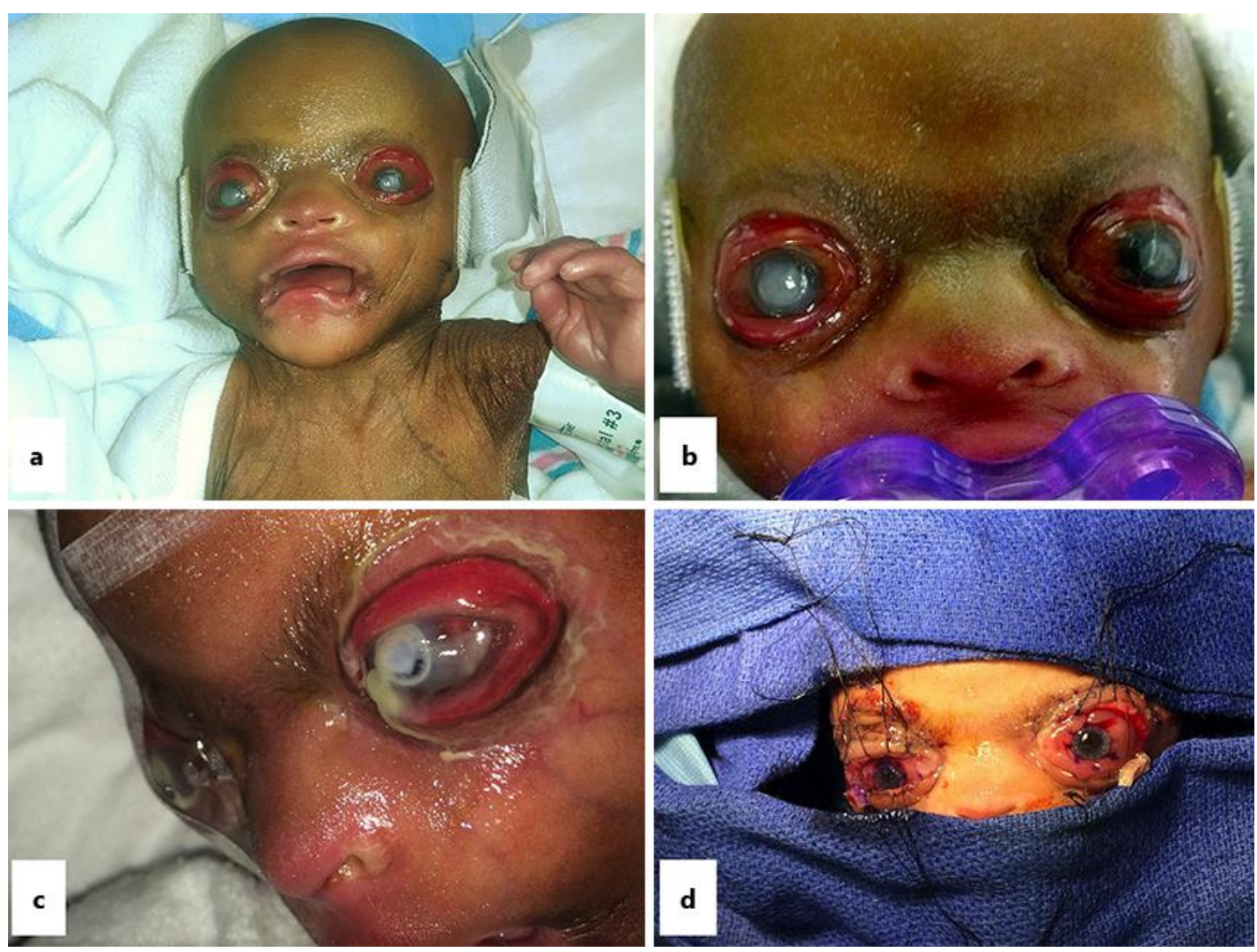

Fig. 1. a, b External photograph of a 5-day-old infant with lack of eyelids, eyelashes and eyebrows. There is significant injection and chemosis in both eyes with bilateral opacification and ulceration of the corneas. Multiple anomalies can be seen on the external photograph including sloping forehead, hypertelorism, flattened nasal bridge, micrognathia, macrostomia, deformed ears, short neck, hyperpigmentation and tense and loose skin in different areas. c External photograph showing significant thinning of the cornea. d External photograph after eyelid reconstruction and bilateral penetrating keratoplasty with clear grafts. 


\section{Case Reports in \\ Ophthalmology}

Feinstein et al.: A Case Report of Ablepharon-Macrostomia Syndrome with Amniotic Membrane Grafting
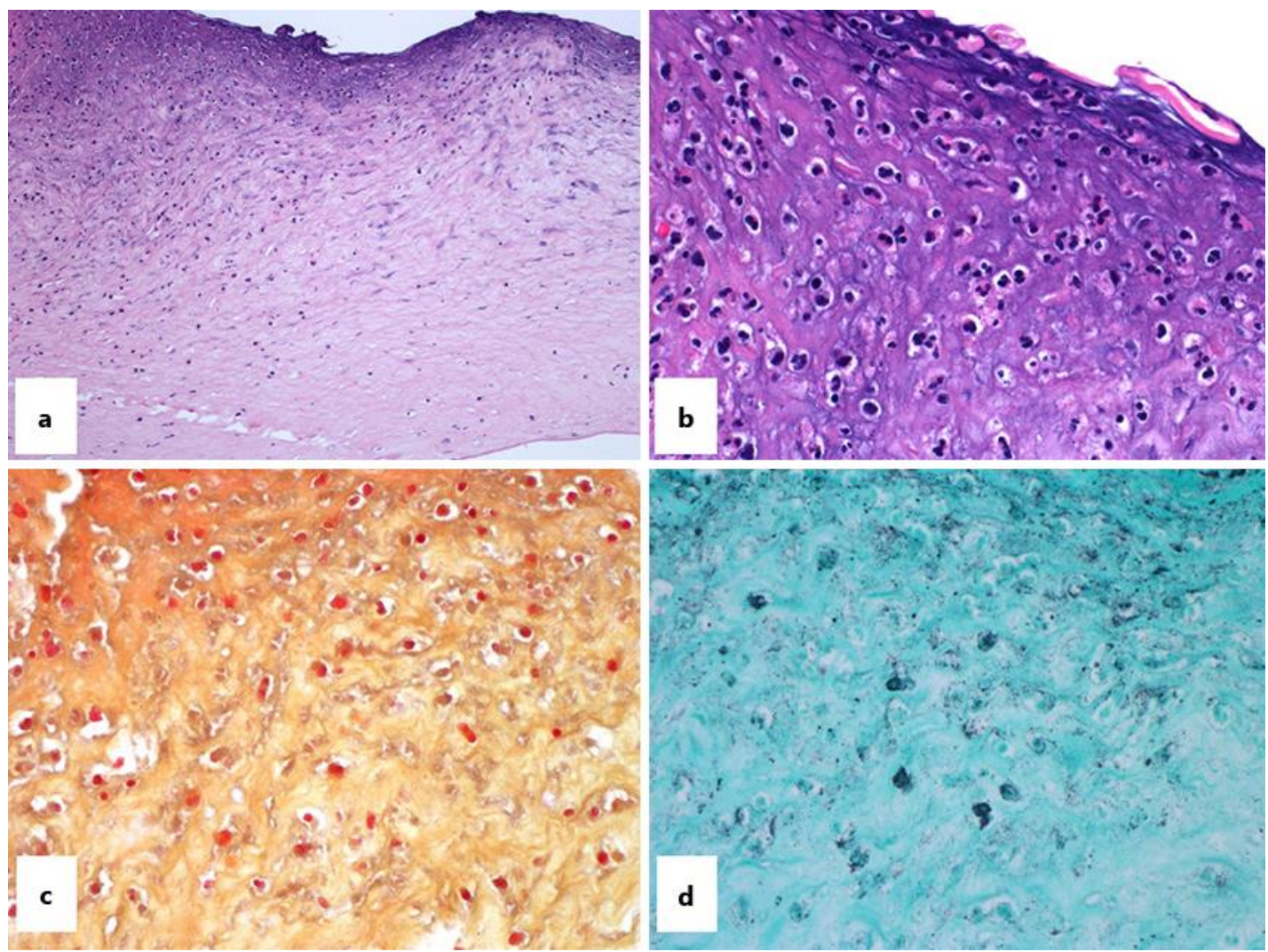

Fig. 2. Corneal pathology slides after full-thickness penetrating keratoplasty OD. a Low-power view of the right cornea with ulceration (top), a neutrophilic infiltrate, and stromal edema. In the bottom right of the image, you can see Descemet's membrane with decreased corneal endothelial cells. b Higher-power field microscopy showing neutrophils in the superficial stroma. c Gram stain which shows no organisms. d Grocott's methenamine silver (GMS) stain negative for fungus both of which are negative (black areas seen on the GMS stain indicate nonspecific background staining). 


\section{Case Reports in \\ Ophthalmology}

\begin{tabular}{l|l}
\hline Case Rep Ophthalmol 2015;6:366-372 \\
\hline DOI: 10.1159/000441615 & $\begin{array}{l}\text { ○ } 2015 \text { The Author(s). Published by S. Karger AG, Basel } \\
\text { www.karger.com/cop }\end{array}$ \\
\hline
\end{tabular}

Feinstein et al.: A Case Report of Ablepharon-Macrostomia Syndrome with Amniotic Membrane Grafting
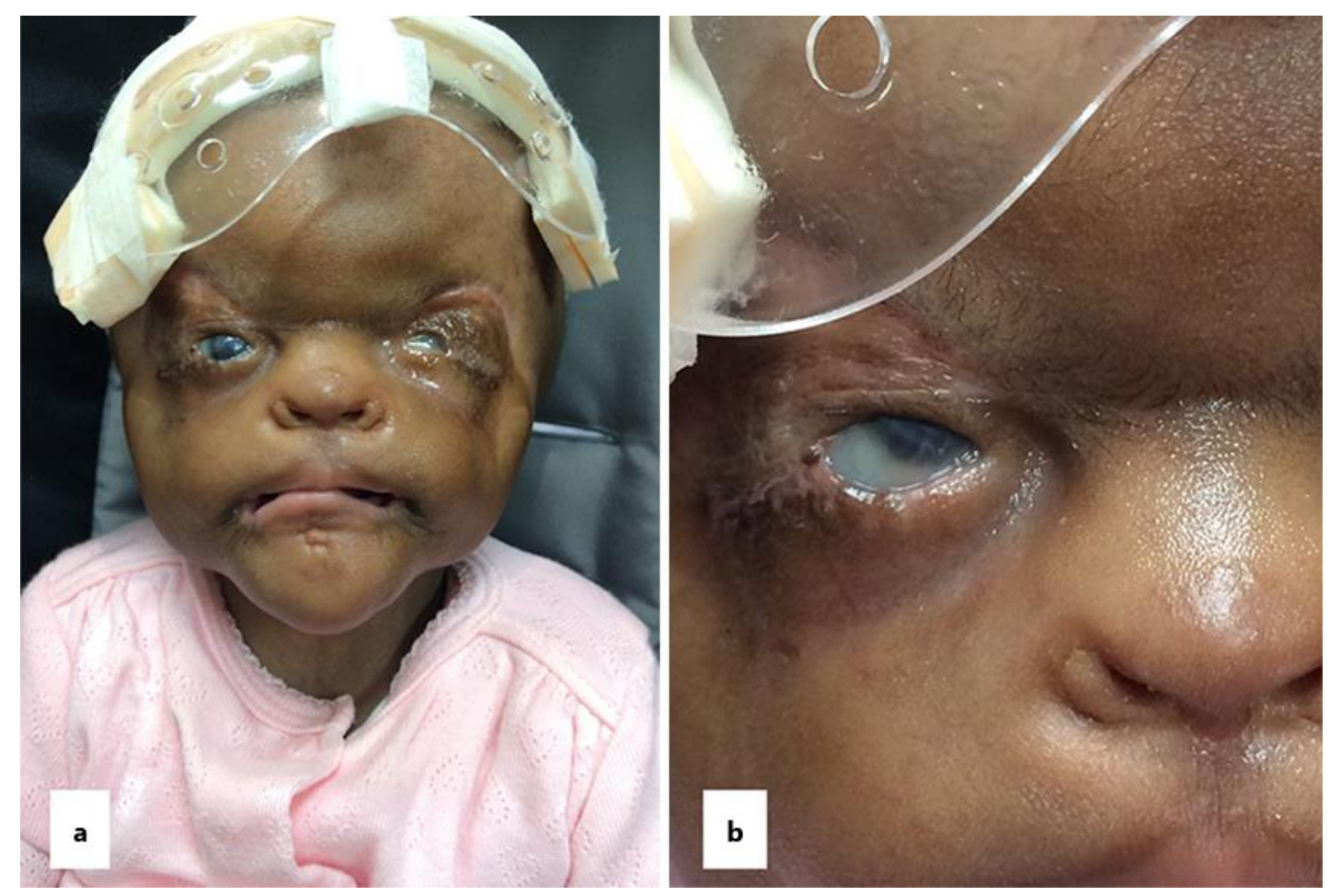

Fig. 3. a, b External color photograph at the age of 5 and a half months. The patient is maintaining good lubrication of the eye with eyelid reconstruction and partial orbicularis function as well as supplemental eyedrops and ointment. 